\title{
Application of artificial intelligence in pediatrics: past, present and future
}

\author{
Li-Qi Shu ${ }^{1} \cdot$ Yi-Kan Sun ${ }^{2} \cdot$ Lin-Hua Tan ${ }^{3} \cdot$ Qiang Shu ${ }^{3} \cdot$ Anthony C. Chang ${ }^{4}$
}

Received: 4 March 2019 / Accepted: 12 March 2019 / Published online: 17 April 2019

(c) Children's Hospital, Zhejiang University School of Medicine 2019

Artificial intelligence (AI) is a very active computer science research field aiming to develop systems that mimic human intelligence and is helpful in many human activities, including medicine. Therefore, it is no surprise that innovation plays an important role in delivering better medical and health.

Initial efforts in AI and its application in medicine began in the 1960s, which focused mainly on diagnosis and therapy. The best-known early works on AI in medicine include Stanford's Ted Shortliffe and his innovative program MYCIN; MYCIN was a rule-based expert system with if-then rules with certainty values that recommended selection of antibiotics for various infectious diseases [1]. Although not used in actual clinical setting, MYCIN was proved to be superior to human infectious disease experts. Szolovits edited a textbook on artificial intelligence in medicine in 1982 with a collection of papers on various topics in this domain [2].

During this early era, academic centers involing AI in medicine included Stanford, MIT, Rutgers, and Pittsburgh in the United States as well as a few centers in Europe. A biennial meeting on artificial intelligence in medicine (The European Society for Artificial Intelligence in Medicine, or AIME) was started in Marseille, France in 1987. Towards the end of the era, Szolovits organized a course on medical artificial intelligence at MIT in 2005 that was one of the

Anthony C. Chang

achang007@aol.com

1 School of Medicine and Health Sciences, George Washington University, Washington, D.C. 20037, USA

2 Faculty of Medicine, University of New South Wales, Sydney, NSW 2052, Australia

3 Children's Hospital, Zhejiang University School of Medicine, Hangzhou 310052, China

4 The Sharon Disney Lund Medical Intelligence and Innovation Institute (MI3), Children's Hospital of Orange County, Orange, CA 92868, USA first organized educational efforts on this burgeoning topic. During this period, popular AI methodologies other than the traditional expert systems included fuzzy logic and neural network, with the latter applied to various clinical situations such as clinical diagnosis and medical images as well as in the critical care setting $[3,4]$.

The emergence of the "trifecta" of: large volumes of available data that required new computational methodologies (or simply Big Data), the escalation of computational power and cloud computing, and the advent of machine learning have promulgated the recent new era of artificial intelligence. Present popular concepts of machine learning include supervised methodologies such as support vector machines, neural networks, and naive Bayesian classifiers, as well as unsupervised techniques such as k-means clustering and principal component analysis. Recent hybrid techniques such as semi-supervised sequence learning can be used with less labeled data. In 2012, the team from University of Toronto used a deep learning algorithm with 650,000 neurons and 5 convolutional layers to reduce the error rate by half during a computer vision challenge. In addition, the IBM supercomputer Watson with its victory in the game show Jeopardy, heralded the era of cognitive computing with its potent natural language processing, knowledge representation and reasoning capabilities. Finally, the DeepMind AlphaGo program defeat of the human Go champion Lee Sedol proved that the computer and deep learning can reach new heights and further advance human understanding in certain topics. The present state of AI in medicine includes a myriad of applications:

\section{Decision support and hospital monitoring}

A recent review of the history of clinical decision support states the dramatic improvement in this sector due to the advent of cognitive aids to support diagnosis, treatment, care-coordination, surveillance and prevention, and health maintenance or wellness [5]. An example of AI application 
in the hospital setting is the use of machine-learning algorithms to automatically classify vital sign alerts as real or artifact, in order to clean such data for future modeling, by training expert-labeled vital sign data streams [6]. Researchers have also studied the creation and implementation of data-driven vital sign parameters to reduce alarm fatigue in a pediatric acute care unit [7]. Another robust illustration of the value of databases coupled with analytics is the Medical Information Mart for Intensive Care (MIMIC) accessible critical care database. MIMIC is of information in the critical care unit from admitted patients for data mining and modeling of conditions. This large, single-center database has resulted in many publications [8].

\section{Medical imaging and biomedical diagnostics}

There is much more promise in the utilization of AI methodologies such as deep learning for automated and/or augmented biomedical image interpretation in radiology, pathology, dermatology, ophthalmology and cardiology. AI techniques including artificial neural networks, support vector machines and classification tree, and ensemble methods such as random forest were applied to molecular imaging modalities in clinical diseases such as neurodegenerative diseases [9]. Sparsifying machine-learning models were used in identifying stable subsets of predictive features for behavioral detection of autism [10]. Machine-learning algorithms were used in the prediction of periventricular leukomalacia in neonates after cardiac surgery [11]. These studies demonstrated that computer-aided diagnosis systems are very promising to contribute to the diagnostic process.

Machine-learning-based methods provide a collection of strategies that are efficient and deliver automated cerebellum parcellations with a high accuracy, surpassing previous work in the area [12]. Machine learning can be used for a number of applications in radiology, including automated detection of disease, segmentation of lesions, and quantitation [13]. In diverse fields of medical image analysis, including nonradiologic tasks like diagnosis of skin lesion and retinal photographs, machine learning can diagnose disease on images at a level comparable to that of skilled physicians. Larson et al. developed and validated a machine-learning system for the assessment of skeletal maturity (i.e., bone age) on pediatric hand radiographs [14].

Google company revealed an algorithm based on deep convolutional neural network to detect diabetic retinopathy in retinal fundus photographs in 2016 [15]. The study showed that the algorithm is as good as board-certified ophthalmologists in making the diagnosis with high specificity and sensitivity and an area under the receiver operating curve of 0.99 .

In biomedical diagnostics, medical geneticists are often frustrated by the tedious nature of genotype-phenotype interrelationships among syndromes, especially for extremely rare syndromes. Now, medical geneticists are able to use a visual diagnostic decision support system that employs machine-learning algorithms and digital imaging processing techniques in a hybrid approach for automated diagnosis in medical genetics [16].

\section{Precision medicine and drug discovery}

Precision medicine with its complexity and enormity of data to be analyzed is particularly well suited for the portfolio of AI methodologies such as deep learning, because similar patients can be assessed and stratified. Precision medicine at its highest level will need a groundbreaking computational platform for new biomedical knowledge discovery.

One such proposal is the BioIntelligence Framework [17]. In this model, a scalable computational framework leverages a hypergraph-based data model and query language that may be suited for representing complex multi-lateral, multi-scalar, and multi-dimensional relationships. This hypergraphlike storage of public knowledge is coupled with an individual's genomic and other patient information (such as imaging data) to drive a personalized genome-based knowledge store for clinical translation and discovery. Patients of very similar genomic and clinical elements can be clustered and matched for diagnostic and therapeutic strategies.

In breast cancer, the combination of computer-aided diagnosis of image-omics and functional genomic features improved the classification accuracy by $3 \%$ [18]. In this study, support vector machine for differentiating stage I breast cancer from other stages is learned with the use of computer-aided diagnosis that enables joint analysis of functional genomic information and image from pathological images. The entire biomedical imaging informatics framework consisted of image extraction, feature combination, and classification.

In addition, there are many potential applications of deep learning for large datasets in pharmaceutical research (such as physicochemical property prediction, formulation prediction, and properties such as absorption, distribution, metabolism, excretion, toxicity, and even target prediction) [19]. An essential part of the precision medicine paradigm is individualized therapy based on genotype-phenotype coupling and pharmacogenomics profiles.

\section{Cloud computing and big data}

Cloud computing in healthcare is at present in the form of singular, individual features such as elasticity, pay-per-use and broad network access rather than as cloud paradigm on its own [20]. Cloud computing is, therefore, often in the 
"OMICS-context" with computing in genomics, proteomics, and molecular medicine with little use in other domains. Although the storage of genomic data into the public cloud raises issues such as security and privacy, initial efforts to secure computation techniques that can enable comparative analysis of human genomes have been productive. One such effort is the NIH-funded National Center for Biomedical Computing iDASH (integrating Data for Analysis, anonymization, and Sharing) and its Critical Assessment of Data Privacy and Protection competition to evaluate the capacity of the cryptographic technologies for protecting computation over human genomes in the cloud while promoting cross-institutional collaboration [21]. In addition, highperformance computing platforms such as clusters, grids and clouds can be used by neurologists, radiologists, and researchers for imaging such as neuroimaging to increase both storage and/or computational power [22].

\section{Digital medicine and wearable technology}

An essential part of digital medicine and wearable devices is the data mining of the incoming data for anomaly detection, prediction, and diagnosis/decision making. The data-mining process for wearable data includes a feature extraction/selection process for modeling/learning to yield detection, prediction, and decision making for the clinician. Expert knowledge and metadata can influence modeling and learning.

The continuously track physiologic parameters ability of wearable devices and sensors can provide an overall patient care strategy for improving outcome and lower healthcare costs in cardiac patients with heart failure [23]. This new paradigm of cardiovascular disease management can also improve the physician-patient relationship. Machine-learning algorithms have also been applied to large-scale wearable sensor data in neurological disorders such as Parkinson's disease to significantly improve both clinical diagnosis and management [24]. This sensor-based, quantitative, objective, and easy-to-use system for assessing Parkinson's disease has potential to replace traditional qualitative and subjective ratings by human interpretation.

\section{Robot technology and virtual assistants}

Surgical robotics such as the da Vinci system has penetrated even community hospitals and has advanced to include 3D visualization. Meanwhile, other uses of robotic technology in healthcare include delivery, sterilization, and physical therapy in various venues. Human-robot interaction is being evaluated and utilized in a variety of clinical scenarios such as rehabilitation and education. There is an ongoing debate about robotics and its ethical implications in the future of society including an exacerbation of healthcare disparities and creation of new ones [25].

In children with autism spectrum disorder, a robot-based approach showed that children with this disorder are more engaged in the several learning tasks and seem to enjoy more the task when interacting with the robot compared with the interaction with the adult [26]. Electromechanical and robot-assisted arm training for improving activities after a stroke showed improvements of these activities in randomized controlled trials. Much is expected of robots and virtual assistants in the future for physical rehabilitation and psychiatric therapy as well as healthcare education and chronic disease management. If accompanied by robust AI tools, these supportive services will be particularly useful in delivering value for the patients.

The future of AI is promising. A myriad of issues in the use of AI remain. One is the ethics of its use in the variety of sectors and the accompanying debates amongst scholars and scientists as well as the public. Another is the computer-human interface and its synergy (or dyssynergy) that remains to be defined as AI evolves. Finally, the eventual possibility of singularity (in which the computers will be more intelligent than all of mankind and replace its intellectual capacity) and how we accommodate this epoch will need to be discussed.

Practitioners require accurate data as well as up-to-date information and sharing of ideas to ensure best outcomes for the patient population. Best practice in the near future will involve the use of data and analytics to answer the clinical questions (intelligence-based medicine) rather than solely relying on published reports currently (evidence-based medicine). It is vital to think of AI as an "energy" (like electricity) rather than as a piece of technological equipment or sophisticated software: in essence, AI can be ubiquitous and invisible in the health care arena and will discover new knowledge from all sources of data and information. The most productive strategy will need to involve an intimate human-machine synergy with a continual clinician-data scientist collaboration.

There are exciting advances in the computer science and AI realm for healthcare and medicine. Quantum computing will be an important advance to accelerate the capabilities of AI tools especially with the vast amounts of data in medicine. Even DNA computing for storage has been suggested to be used to increase storage capacity. In addition, cloud computing and storage will be vital to facilitate the panoply of AI techniques for multi-institutional collaborations that will be essential for the future of AI in biomedicine and healthcare. The internet of things and everything (IoT and IoE) will also provide the critical data sources for medicine in the form of wearable and monitoring devices from both hospital and home. Finally, the full promise of AI in medicine will require a data security and privacy transformation (via disruptive technology such as blockchain) and 
an innovation focus from regulatory agencies and software vendors (via open source collaboration).

Author contributions LQS and YKS contributed equally to this work. LQS and YKS drafted the initial manuscript. QS, LHT and ACC reviewed, revised, and approved the final manuscript.

Funding LHT received support for article research from the Natural Science Foundation of China through grant 81571475 and Health Bureau of Zhejiang Province through grant 2010KYA122. QS received support from the Natural Science Foundation of China through grant 81671956 and Zhejiang Provincial Program for the Cultivation of High-level Innovative Health Talents Program.

\section{Compliance with ethical standards}

Ethical approval Not needed.

Conflict of interest The authors have no financial relationships relevant to this article to disclose.

\section{References}

1. Shortliffe EH, Davis R, Axline SG, Buchanan BG, Green CC, Cohen SN. Computer-based consultations in clinical therapeutics: explanation and rule acquisition capabilities of the MYCIN system. Comput Biomed Res. 1975;8:303-20.

2. Szolovits P. Artificial intelligence in medicine. Boulder: Westview Press Inc; 1982.

3. Ramesh AN, Kambhampati C, Monson JR, Drew PJ. Artificial intelligence in medicine. Ann R Coll Surg Engl. 2004;86:334-8.

4. Hanson CW 3rd, Marshall BE. Artificial intelligence applications in the intensive care unit. Crit Care Med. 2001;29:427-35.

5. Middleton B, Sittig DF, Wright A. Clinical decision support: a 25 year retrospective and a 25 year vision. Yearb Med Inform. 2016;Suppl 1:S103-16.

6. Hravnak M, Chen L, Dubrawski A, Bose E, Clermont G, Pinsky MR. Real alerts and artifact classification in archives multi-signal vital sign monitoring data: implication for mining big data. J Clin Monit Comput. 2016;30:875-88.

7. Goel VV, Poole SF, Longhurst CA, Platchek TS, Pageler NM, Sharek PJ, et al. Safety analysis of proposed data-driven physiologic alarm parameters for hospitalized children. J Hosp Med. 2016;11:817-23.

8. Johnson AE, Pollard TJ, Shen L, Lehman LW, Feng M, Ghassemi $\mathrm{M}$, et al. MIMIC-III, a freely accessible critical care database. Sci Data. 2016;3:160035.

9. Cascianelli S, Scialpi M, Amici S, Forini N, Minestrini M, Fravolini ML, et al. Role of artificial intelligence techniques (Automatic Classifiers) in molecular imaging modalities in neurodegenerative disease. Curr Alzheimer Res. 2017;14:198-207.
10. Levy S, Duda M, Haber N, Wall DP. Sparsifying machine learning models identify stable subsets of predictive features for behavioral detection of autism. Mol Autism. 2017;8:65.

11. Jalali A, Simpao AF, Gálvez JA, Licht DJ, Nataraj C. Prediction of periventricular leukomalacia in neonates after cardiac surgery using machine learning algorithms. J Med Syst. 2018;42:177.

12. Carass A, Cuzzocreo JL, Han S, Hernandez-Castillo CR, Rasser PE, Ganz M, et al. Comparing fully automated state-of-the-art cerebellum parcellation from magnetic resonance images. Neuroimage. 2018;183:150-72.

13. Summers RM. Deep learning lends a hand to pediatric radiology. Radiology. 2018;287:323-5.

14. Larson DB, Chen MC, Lungren MP, Halabi SS, Stence NV, Langlotz CP. Performance of a deep-learning neural network model in assessing skeletal maturity on pediatric hand radiographs. Radiology. 2018;287:313-22.

15. Gulshan V, Peng L, Coram M, Stumpe MC, Wu D, Narayanaswamy A, et al. Development and validation of a deep learning algorithm for detection of diabetic retinopathy in retinal fundus photographs. JAMA. 2016;316:2402-10.

16. Kuru K, Niranjan M, Tunca Y, Osvank E, Azim T. Biomedical visual data analysis to build an intelligent diagnostic decision support system in medical genetics. Artif Intell Med. 2014;62:105-18.

17. Farley T, Kiefer J, Lee P, Von Hoff D, Trent JM, Colbourn C, et al. The BioIntelligence Framework: a new computational platform for biomedical knowledge computing. J Am Med Inform Assoc. 2013;20:128-33.

18. Su H, Shen Y, Xing F, Qi X, Hirshfield KM, Yang L, et al. Robust automatic breast cancer staging using a combination of functional genomics and image-omics. Conf Proc IEEE Eng Med Biol Soc. 2015;2015:7226-9.

19. Ekins $S$. The next era: deep learning in pharmaceutical research. Pharm Res. 2016;33:2594-603.

20. Griebel L, Prokosch HU, Kopcke F, Toddenroth D, Christoph J, Leb I, et al. A scoping review of cloud computing in healthcare. BMC Med Inform Decis Mak. 2015;15:17.

21. Tang H, Jiang X, Wang X, Wang S, Sofia H, Fox D, et al. Protecting genomic data analytics in the cloud: state of the art and opportunities. BMC Med Genom. 2016;9:63.

22. Shatil AS, Younas S, Pourreza H, Figley CR. Heads in the cloud: a primer on neuroimaging applications of the high performance computing. Magn Reson Insights. 2016;8(Suppl 1):69-80.

23. Steinhubl SR, Topol EJ. Moving from digitalization to digitization in cardiovascular care: why is it important, and what could it mean for patients and providers? J Am Coll Cardiol. 2015;66:1489-96.

24. Kubota KJ, Chen JA, Little MA. Machine learning for large-scale wearable sensor data in Parkinson's disease: concepts, promises, pitfalls, and features. Mov Disord. 2016;31:1314-26.

25. Russell S, Hauert S, Altman R, Veloso M. Robotics: ethics of artificial intelligence. Nature. 2015;521:415-8.

26. Costescu CA, Vanderborght B, David DO. Reversal learning task in children with autism spectrum disorder: a robot-based approach. J Autism Dev Disord. 2015;45:3715-25.

Publisher's Note Springer Nature remains neutral with regard to jurisdictional claims in published maps and institutional affiliations. 